\title{
On a method of construction of new means with applications
}

\author{
Mustapha Raïssouli ${ }^{1,2^{*}}$ and József Sándor ${ }^{3}$
}

\section{"Correspondence:}

raissouli_10@hotmail.com

${ }^{1}$ Faculty of Science, Department of Mathematics, Taibah University, P.O.

Box 30097, Al Madinah Al

Munawwarah, Zip Code 41477,

Kingdom of Saudi Arabia

${ }^{2}$ Faculty of Sciences, Department of

Mathematics, Moulay Ismail

University, Meknes, Morocco

Full list of author information is

available at the end of the article

\begin{abstract}
In the present paper, we would like to introduce a simple transformation for bivariate means from which we derive a lot of new means. Relationships between the standard means are also obtained. A simple link between the Stolarsky mean and the Gini mean is given. As applications, this transformation allows us to extend some means from two to three or more arguments.
\end{abstract}

MSC: $26 \mathrm{E} 60$

Keywords: means; decomposable means; mean-inequalities; Stolarsky mean; Gini mean

\section{Introduction and basic notions}

In the recent past, the theory of means has been the subject intensive research. It has proved to be a useful tool for theoretical viewpoint as well as for practical purposes. For the definition of a mean, various statements, more or less different, can be found in the literature; see [1] and the references therein. Throughout this paper, we adopt the following definition.

Definition 1.1 A function $m:(0, \infty) \times(0, \infty) \longrightarrow(0, \infty)$ is called a mean if

$$
\forall a, b>0 \quad \min (a, b) \leq m(a, b) \leq \max (a, b) .
$$

From this, it is clear that every mean is with positive values and reflexive, that is, $m(a, a)=$ $a$ for each $a>0$. The maps $(a, b) \longmapsto \min (a, b)$ and $(a, b) \longmapsto \max (a, b)$ are (trivial) means which will be denoted by min and max, respectively. The standard examples of means are given as follows:

$$
\begin{aligned}
& A:=A(a, b)=\frac{a+b}{2} ; \quad G:=G(a, b)=\sqrt{a b} ; \quad H:=H(a, b)=\frac{2 a b}{a+b} ; \\
& L:=L(a, b)=\frac{b-a}{\ln b-\ln a}, \quad L(a, a)=a ; \\
& I:=I(a, b)=\frac{1}{e}\left(\frac{b^{b}}{a^{a}}\right)^{1 /(b-a)}, \quad I(a, a)=a ; \\
& S:=S(a, b)=a^{a /(a+b)} b^{b /(a+b)} ; \quad C:=C(a, b)=\frac{a^{2}+b^{2}}{a+b} ; \\
& P:=P(a, b)=\frac{b-a}{4 \arctan \sqrt{b / a}-\pi}=\frac{b-a}{2 \arcsin \frac{b-a}{b+a}}, \quad P(a, a)=a
\end{aligned}
$$

@ 2013 Raïssouli and Sándor; licensee Springer. This is an Open Access article distributed under the terms of the Creative Commons Attribution License (http://creativecommons.org/licenses/by/2.0), which permits unrestricted use, distribution, and reproduction in any medium, provided the original work is properly cited. 
and are known as the arithmetic, geometric, harmonic, logarithmic, identric, weighted geometric, contraharmonic and (first) Seiffert means, respectively.

The set of all means can be equipped with a partial ordering, called a point-wise order, defined by $m_{1} \leq m_{2}$ if and only if $m_{1}(a, b) \leq m_{2}(a, b)$ for every $a, b>0$. We write $m_{1}<m_{2}$ if and only if $m_{1}(a, b)<m_{2}(a, b)$ for all $a, b>0$ with $a \neq b$. With this, the above means satisfy the known chain of inequalities

$\min <H<G<L<P<I<A<S<C<\max$.

A mean $m$ is symmetric if $m(a, b)=m(b, a)$ and homogeneous if $m(t a, t b)=\operatorname{tm}(a, b)$ for all $a, b, t>0$. The above means are all symmetric and homogeneous. However, the mean $(a, b) \longmapsto(2 a+b) / 3$ is (homogeneous) not symmetric, while $(a, b) \longmapsto \ln \left(\frac{e^{a}+e^{b}}{2}\right)$ is (symmetric) not homogeneous. The mean $(a, b) \longmapsto \ln \left(\frac{2 e^{a}+e^{b}}{3}\right)$ is neither symmetric nor homogeneous.

A mean $m$ is called monotone if $(a, b) \longmapsto m(a, b)$ is increasing in $a$ and in $b$, that is, if $a_{1} \leq a_{2}$ (resp. $\left.b_{1} \leq b_{2}\right)$, then $m\left(a_{1}, b\right) \leq m\left(a_{2}, b\right)$ (resp. $\left.m\left(a, b_{1}\right) \leq m\left(a, b_{2}\right)\right)$. There are many means which are not monotone. For example, it is easy to see that the means $A, G, H, L$ are monotone but $C$ is not. However, extending the above definitions of reflexivity and monotonicity from a mean to a general binary map, the following result is of interest $[2$, $3]$.

Proposition 1.1 Let $m$ be a monotone and reflexive map. Then $m$ is a mean.

For a given mean $m$, we set $m^{*}(a, b)=\left(m\left(a^{-1}, b^{-1}\right)\right)^{-1}$, and it is easy to see that $m^{*}$ is also a mean, called the dual mean of $m$. If $m$ is homogeneous, then so is $m^{*}$ with $m^{*}(a, b)=$ $a b / m(b, a)$. If $m$ is symmetric and homogeneous, then so is $m^{*}$, and in this case, we have $m^{*}(a, b)=a b / m(a, b)$. Every mean $m$ satisfies $m^{* *}:=\left(m^{*}\right)^{*}=m$, and if $m_{1}$ and $m_{2}$ are two means such that $m_{1} \leq m_{2}$, then $m_{1}^{*} \geq m_{2}^{*}$. One can check that $\min ^{*}=\max$ and $\max { }^{*}=\min$. Further, the arithmetic and harmonic means are mutually dual (i.e., $A^{\prime \prime}=H, H^{*}=A$ ) and the geometric mean is self-dual (i.e., $G^{*}=G$ ). The dual of the logarithmic and identric means has been studied by the second author in [4].

The following inequalities are immediate from the above:

$$
\min <C^{*}<S^{*}<H<I^{*}<P^{*}<L^{*}<G<L<P<I<A<S<C<\max .
$$

Remark 1.1 Let $k:] 0,+\infty[\longrightarrow] 0,+\infty$ [ be a monotone continuous function and denote by $k^{-1}$ its inverse function. An extension of the dual mean can be given by $m_{k}(a, b)=$ $k^{-1}(m(k(a), k(b)))$. It is easy to verify that $m_{k}$ is a mean. For $k(x)=1 / x$, we obtain the classical dual. If we choose $k(x)=x^{-r}$ with $r>0$, then we get the following generalized dual $m^{*} r(a, b)=\left(m\left(a^{-r}, b^{-r}\right)\right)^{-1 / r}$. If $m$ is symmetric and homogeneous, then $m^{*} r(a, b)=$ $a b /\left(m\left(a^{r}, b^{r}\right)\right)^{1 / r}$.

Let $m$ be a homogeneous mean. Writing $m(a, b)=b m(a / b, 1)$, we then associate to $m$ a unique positive function $f$ defined by $f(x)=m(x, 1)$ for all $x>0$. The function $f$ will be called the associated function to the mean $m$, or we simply say that $f$ corresponds to the mean $m$. It follows that $f$ corresponds to a homogeneous mean if and only if $\min (x, 1) \leq$ 
$f(x) \leq \max (x, 1)$. Clearly, $f(1)=1$, and if, moreover, $m$ is symmetric, then $f(x)=x f(1 / x)$ for every $x>0$. It is obvious that a mean $m$ is monotone if and only if its associated function is increasing. For example, the contraharmonic mean $C$ is not monotone because its associated function $f(x)=\left(x^{2}+1\right) /(x+1)$ satisfies $(x+1)^{2} f^{\prime}(x)=x^{2}+2 x-1$, and it is easy to see that $f$ is not increasing for all $x>0$, but only for $x>\sqrt{2}-1$.

Now, let us observe the next question : under which sufficient condition a given function $f$ is the associated function of a certain mean? The following result gives an answer to this situation; see [5], Remark 12.

Proposition 1.2 Let $f:] 0,+\infty[\longrightarrow] 0, \infty[$ be a function such that $\min (x, 1) \leq f(x) \leq$ $\max (x, 1)$ for every $x>0$. Then $m(a, b)=b f(a / b)$ defines $a$ (homogeneous) mean. If, moreover, $f$ is increasing with $f(x)=x f(1 / x)$ for each $x>0$, then $m$ is monotone symmetric.

The next result is also of interest to our present paper; see [2, 3].

Proposition 1.3 Let $m$ be a symmetric and homogeneous mean having a strictly increasing associated function $f$. Then $f^{*}$, the associated function to the dual mean $m^{*}$, is strictly increasing, too.

By virtue of the relation $m^{* * *}=m$ (and so $f^{* * *}=f$ ), the result of the above proposition is in fact an equivalence. It follows that the associated function of the dual $C^{*}$ will be not always increasing since that of $C$ is not.

In the literature, there are some families of means which include the above familiar means. Precisely, let $p$ and $q$ be two real numbers. The Stolarsky mean $E_{p, q}$ of order $(p, q)$ is defined for all $a, b>0$ such that $a \neq b$ as well by

$$
E_{p, q}(a, b):= \begin{cases}\left(\frac{p}{q} \frac{b^{q}-a^{q}}{b^{p}-a^{p}}\right)^{1 /(q-p)} & \text { if } p q(p-q) \neq 0, \\ \exp \left(-\frac{1}{p}+\frac{a^{p} \ln a-b^{p} \ln b}{a^{p}-b^{p}}\right) & \text { if } p=q \neq 0, \\ \left(\frac{1}{p} \frac{b^{p}-a^{p}}{\ln b-\ln a}\right)^{1 / p} & \text { if } p \neq 0, q=0, \\ \sqrt{a b} & \text { if } p=q=0\end{cases}
$$

with $E_{p, q}(a, a)=a$, while the Gini mean $G_{p, q}$ of order $(p, q)$ is defined by

$$
G_{p, q}(a, b):= \begin{cases}\left(\frac{a^{q}+b^{q}}{a^{p}+b^{p}}\right)^{1 /(q-p)} & \text { if } p \neq q \\ \exp \left(\frac{a^{p} \ln a+b^{p} \ln b}{a^{p}+b^{p}}\right) & \text { if } p=q \neq 0 \\ \sqrt{a b} & \text { if } p=q=0\end{cases}
$$

Clearly, the means $E_{p, q}$ and $G_{p, q}$ are symmetric and homogeneous. Further, $E_{p, q}$ and $G_{p, q}$ are symmetric in $p$ and $q$. It is worth mentioning that

$$
E_{p, 2 p}(a, b)=G_{0, p}(a, b)= \begin{cases}\left(\frac{a^{p}+b^{p}}{2}\right)^{1 / p} & \text { if } p \neq 0 \\ \sqrt{a b} & \text { if } p=0\end{cases}
$$

is called the power mean of order $p$. It is well known that $E_{p, q}$ and $G_{p, q}$ are strictly increasing with both $p$ and $q$. For particular choices of $p$ and $q$, we find again

$$
E_{-2,-1}=H<E_{0,0}=G<E_{1,0}=L<E_{1,1}=I<E_{2,1}=G_{0,1}=A<G_{1,1}=S<G_{2,1}=C .
$$




\section{A mean-transformation}

The following formula

$$
L(a, b)=\prod_{n=1}^{\infty} A\left(a^{1 / 2^{n}}, b^{1 / 2^{n}}\right):=\prod_{n=1}^{\infty} \frac{a^{1 / 2^{n}}+b^{1 / 2^{n}}}{2}
$$

is well known in the literature [1]; see also [6, 7] for another approach. The aim of this section is to observe (1) in a general point of view. The second side of (1) is an infinite product involving the arithmetic mean $A$ and the geometric sequence $\left(1 / 2^{n}\right)$. Let us try to replace $A$ by an arbitrary mean $m$ and $\left(1 / 2^{n}\right)$ by a general sequence. Two points arise from this situation: the corresponding infinite product should be convergent and we need its value to define a mean. By virtue of the double inequality

$$
\min (a, b) \leq m(a, b) \leq \max (a, b)
$$

which is valid for every mean $m$, the infinite product $\prod_{n=1}^{\infty} m\left(a^{t_{n}}, b^{t_{n}}\right)$ will be convergent provided that the sequence $\left(t_{n}\right)$ has a constant sign and the series $\sum_{n=1}^{\infty} t_{n}$ is convergent. We must choose $\left(t_{n}\right)$ positive and satisfying $\sum_{n=1}^{\infty} t_{n}=1$, and so we have

$$
\min (a, b) \leq \prod_{n=1}^{\infty} m\left(a^{t_{n}}, b^{t_{n}}\right) \leq \max (a, b) .
$$

Summarizing the above, we may state the following.

Definition 2.1 Let $t=\left(t_{n}\right)_{n \geq 1}$ be a positive sequence such that $\sum_{n=1}^{\infty} t_{n}=1$ and $m$ be a mean. For all $a, b>0$, define

$$
m^{\pi_{t}}(a, b)=\prod_{n=1}^{\infty} m\left(a^{t_{n}}, b^{t_{n}}\right)
$$

which we call the $t$-transformation of $m$.

We explicitly notice that the convergence of the infinite product in (3) is shown by the double inequality (2).

The elementary properties of the mean-transformation $m \rightarrow m^{\pi t}$ are summarized in the next result.

Proposition 2.1 Let $m, m_{1}, m_{2}$ be given means and $t=\left(t_{n}\right)$ be a positive sequence such that $\sum_{n=1}^{\infty} t_{n}=1$. Then the following assertions are fulfilled:

(i) $m^{\pi_{t}}$ is a mean.

(ii) If $m$ is homogeneous (resp. symmetric, monotone), then so is $m^{\pi_{t}}$ and the associated function $f^{\pi_{t}}$ to $m^{\pi_{t}}$ is given by

$$
\forall x>0 \quad f^{\pi_{t}}(x)=\prod_{n=1}^{\infty} f\left(x^{t_{n}}\right),
$$

where $f$ is the associated function to $\mathrm{m}$. 
(iii) $\left(m^{\pi_{t}}\right)^{\prime s}=\left(m^{\prime \prime}\right)^{\pi_{t}}$ for each $s>0$, where ${ }^{*}$ denotes the generalized dual mean (see Remark 1.1).

(iv) $m_{1}<m_{2} \Longrightarrow m_{1}^{\pi t}<m_{2}^{\pi t}$.

(v) $\forall \alpha \in[0,1]\left(m_{1}^{1-\alpha} m_{2}^{\alpha}\right)^{\pi_{t}}=\left(m_{1}^{\pi_{t}}\right)^{1-\alpha}\left(m_{2}^{\pi_{t}}\right)^{\alpha}$.

\section{Proof}

(i) It is already proved by (2).

(ii) The symmetry of $m^{\pi_{t}}$ from that of $m$ is obvious, while the monotonicity of $m^{\pi_{t}}$ follows from the fact that $m$ is monotone and $m^{\pi_{t}}$ is defined as (infinite) product of positive terms. Now, assume that $m$ is homogeneous. By definition, we have for all $a, b, \alpha>0$

$$
m^{\pi_{t}}(\alpha a, \alpha b)=\prod_{n=1}^{\infty} m\left((\alpha a)^{t_{n}},(\alpha b)^{t_{n}}\right)=\prod_{n=1}^{\infty} \alpha^{t_{n}} \prod_{n=1}^{\infty} m\left(a^{t_{n}}, b^{t_{n}}\right) .
$$

The homogeneity of $m^{\pi_{t}}$ follows since $\prod_{n=1}^{\infty} \alpha^{t_{n}}=\alpha^{\sum_{n=1}^{\infty} t_{n}}=\alpha$.

The homogeneity of $m$ and $m^{\pi_{t}}$ implies, with (3), that the associated function of $m^{\pi_{t}}$ is given by

$$
\forall x>0 \quad f^{\pi_{t}}(x)=m^{\pi_{t}}(x, 1)=\prod_{n=1}^{\infty} m\left(x^{t_{n}}, 1\right)=\prod_{n=1}^{\infty} f\left(x^{t_{n}}\right) .
$$

(iii) By definition, we have successively

$$
\begin{aligned}
\left(m^{\pi t}\right)^{* s}(a, b) & =\left(m^{\pi t}\left(a^{-s}, b^{-s}\right)\right)^{-1 / s}=\left(\prod_{n=1}^{\infty} m\left(a^{-s t_{n}}, b^{-s t_{n}}\right)\right)^{-1 / s} \\
& =\prod_{n=1}^{\infty}\left(m\left(\left(a^{t_{n}}\right)^{-s},\left(b^{t_{n}}\right)^{-s}\right)\right)^{-1 / s}=\prod_{n=1}^{\infty} m^{* s}\left(a^{t_{n}}, b^{t_{n}}\right)=\left(m^{*} s\right)^{\pi_{t}} .
\end{aligned}
$$

(iv) and (v) are not difficult. Details are omitted for the reader as a simple exercise.

We now present the following examples. In all these examples, the sequence $t=\left(t_{n}\right)$ is as in the above.

Example 2.1 It is easy to verify that $G^{\pi_{t}}=G$. Otherwise, we have

$$
A^{\pi_{t}}(a, b)=\prod_{n=1}^{\infty} \frac{a^{t_{n}}+b^{t_{n}}}{2} \leq \prod_{n=1}^{\infty}\left(\frac{a+b}{2}\right)^{t_{n}}=\frac{a+b}{2}=A(a, b),
$$

with strict inequality for $a \neq b$ since $0<t_{n}<1$ and the map $x \longmapsto x^{\alpha}$ is strictly concave for $0<\alpha<1$. We then have $A^{\pi_{t}}<A$, and by Proposition 1.1(iii) and (iv), we deduce $H<H^{\pi_{t}}$. In summary, we have

$$
H<H^{\pi_{t}}<G^{\pi_{t}}=G<A^{\pi_{t}}<A .
$$

Example 2.2 According to the definition, with (1), we have

$$
L^{\pi_{t}}(a, b)=\prod_{n=1}^{\infty} L\left(a^{t_{n}}, b^{t_{n}}\right)=\prod_{n=1}^{\infty} \prod_{i=1}^{\infty} \frac{a^{t_{n} / 2^{i}}+b^{t_{n} / 2^{i}}}{2} \leq \prod_{i=1}^{\infty} \prod_{n=1}^{\infty}\left(\frac{a^{1 / 2^{i}}+b^{1 / 2^{i}}}{2}\right)^{t_{n}}
$$


for the same reason as in the above example. Then

$$
L^{\pi_{t}}(a, b) \leq \prod_{i=1}^{\infty}\left(\frac{a^{1 / 2^{i}}+b^{1 / 2^{i}}}{2}\right)^{\sum_{n=1}^{\infty} t_{n}}=\prod_{i=1}^{\infty} \frac{a^{1 / 2^{i}}+b^{1 / 2^{i}}}{2}=L(a, b) .
$$

Further, the above inequalities are strict for $a \neq b$. It follows that $L^{\pi_{t}}<L$ and so

$$
L^{*}<L^{*} \pi_{t}<G^{\pi_{t}}=G<L^{\pi_{t}}<L .
$$

Example 2.3 It is well known that

$$
I(a, b)=\exp \int_{0}^{1} \log ((1-s) a+s b) d s .
$$

With this we have

$$
\begin{aligned}
I^{\pi_{t}}(a, b) & =\prod_{n=1}^{\infty}\left(\exp \int_{0}^{1} \log \left((1-s) a^{t_{n}}+s b^{t_{n}}\right) d s\right) \\
& =\exp \left(\sum_{n=1}^{\infty} \int_{0}^{1} \log \left((1-s) a^{t_{n}}+s b^{t_{n}}\right) d s\right) \\
& \leq \exp \left(\sum_{n=1}^{\infty} \int_{0}^{1} \log ((1-s) a+s b)^{t_{n}} d s\right) .
\end{aligned}
$$

We deduce that $I^{\pi_{t}}(a, b) \leq I(a, b)$ with strict inequality for $a \neq b$. It follows that $I^{\pi_{t}}<I$ and so

$$
I^{*}<I^{*} \pi_{t}<G^{\pi_{t}}=G<I^{\pi_{t}}<I .
$$

Reduction of the three chains of inequalities (4), (5) and (6) in one chain does not appear to be obvious. However, for the particular case $t_{n}=1 / 2^{n}$, the above three chains can be reduced into one chain; see Corollary 4.1 in Section 4 below.

Example 2.4 Let $r$ be a real number such that $0<r<1$. Setting $t_{n}=(1-r) r^{n-1}$, we have $\sum_{n=1}^{\infty} t_{n}=1$, and so the hypotheses of the above definition are satisfied. In this case, we write

$$
m^{\pi_{t}}(a, b):=m^{\pi_{r}}(a, b)=\prod_{n=1}^{\infty} m\left(a^{(1-r) r^{n-1}}, b^{(1-r) r^{n-1}}\right) .
$$

The situation of this example will be developed below.

In what precedes, starting from a given mean $m$, we have defined a new class of means $m^{\pi_{t}}$ provided that the positive sequence $t=\left(t_{n}\right)$ satisfies $\sum_{n=1}^{\infty} t_{n}=1$. Our procedure can be recursively continued: for $k$ positive sequences $t_{1}=\left(t_{n, 1}\right), t_{2}=\left(t_{n, 2}\right), \ldots, t_{k}=\left(t_{n, k}\right)$ such that $\sum_{n=1}^{\infty} t_{n, i}=1$ for $i=1,2, \ldots, k$, we can define the following:

$$
m^{\pi} T_{k}=\left(m^{\pi} T_{k-1}\right)^{\pi t_{k}}
$$


where we set $T_{k}:=\left(t_{1}, t_{2}, \ldots, t_{k}\right)$, or explicitly,

$$
m^{\pi T_{k}}(a, b)=\prod_{n=1}^{\infty} m\left(a^{T_{n, k}}, b^{T_{n, k}}\right)
$$

with $T_{n, k}:=\prod_{i=1}^{k} t_{n, i}$. In particular, if $t_{n, i}=\left(1-r_{i}\right) r_{i}^{n-1}$ with $0<r_{i}<1$ for $i=1,2, \ldots, k$, we obtain

$$
m^{\pi_{R_{k}}}(a, b)=\prod_{n=1}^{\infty} m\left(a^{R_{n, k}}, b^{R_{n, k}}\right)
$$

where we put

$$
R_{k}:=\left(r_{1}, r_{2}, \ldots, r_{k}\right) \quad \text { and } \quad R_{n, k}:=\prod_{i=1}^{k}\left(1-r_{i}\right) \cdot\left(\prod_{i=1}^{k} r_{i}\right)^{n-1} \text {. }
$$

In what follows, we will explore this latter situation in more detail. For the sake of simplicity, we restrict ourselves to the case $r=1 / 2$. The general case can be stated in a similar manner and we leave it to the reader. Precisely, we put the following.

Definition 2.2 Let $m$ be a given mean. We set $m^{\pi_{0}}=m, m^{\pi_{1}}=m^{\pi}$ and, for all integer $k \geq 0$,

$$
m^{\pi_{k+1}}=\left(m^{\pi_{k}}\right)^{\pi}=\left(m^{\pi}\right)^{\pi_{k}}
$$

Clearly, $m^{\pi_{k}}$ is a mean for all $k \geq 0$. The next example may be stated.

Example 2.5 We have $G^{\pi_{k}}=G$ for each $k \geq 0$. Formula (1) written in a brief form $L=A^{\pi}$, with a mathematical induction, yields

$$
L^{\pi_{k-1}}(a, b)=A^{\pi_{k}}(a, b)=\prod_{n_{1}, n_{2}, \ldots, n_{k}=1}^{\infty} A\left(a^{1 / 2^{N_{k}}}, a^{1 / 2^{N_{k}}}\right),
$$

where we put $N_{k}:=\sum_{i=1}^{k} n_{i}$ for every $k \geq 1$.

If we apply the above definition to the standard means, we obtain the following iterative inequalities:

$$
G<L^{\pi_{k}}<P^{\pi_{k}}<I^{\pi_{k}}<A^{\pi_{k}}=L^{\pi_{k-1}}<P^{\pi_{k-1}}<I^{\pi_{k-1}}<A^{\pi_{k-1}}=L^{\pi_{k-2}}<\cdots .
$$

A sequence $\left(m_{k}\right)_{k}$ of means will be called point-wise convergent (in short $p$-convergent) if, for all $a, b>0$, the real sequence $\left(m_{k}(a, b)\right)_{k}$ converges. Setting $m_{\infty}(a, b)=\lim _{k} m_{k}(a, b)$, it is easy to see that $m_{\infty}$ is a mean. Similarly, we define the point-wise monotonicity of $\left(m_{k}\right)_{k}$. By virtue of the double inequality

$$
\min (a, b) \leq m_{k}(a, b) \leq \max (a, b),
$$


we deduce that every $p$-increasing (resp. $p$-decreasing) sequence $\left(m_{k}\right)_{k}$ is $p$-convergent. This together with inequalities (7) implies that the mean-sequences $\left(L^{\pi_{k}}\right)_{k},\left(P^{\pi_{k}}\right)_{k},\left(I^{\pi_{k}}\right)_{k}$ and $\left(A^{\pi_{k}}\right)_{k}$ are decreasingly $p$-convergent. Then, what are their limits? The answer to this latter question will be presented in the next section after stating some needed results.

\section{Study of a special case}

As already pointed before, this section will be devoted to studying the family of means $m^{\pi t}$ for the case $t_{n}=(1-r) r^{n-1}$ with $0<r<1$. We recall the following formula:

$$
m^{\pi_{t}}(a, b):=m^{\pi_{r}}(a, b)=\prod_{n=1}^{\infty} m\left(a^{(1-r) r^{n-1}}, b^{(1-r) r^{n-1}}\right)
$$

which we call the $r$-decomposition of $m$. If $r=1 / 2$, we simply write $m^{\pi}$, that is,

$$
m^{\pi}(a, b):=\prod_{n=1}^{\infty} m\left(a^{1 / 2^{n}}, b^{1 / 2^{n}}\right)
$$

In what follows, we will see that the mean $m^{\pi_{r}}$ satisfies good properties, the first of which is announced as well.

Proposition 3.1 With the above, the following assertions hold true:

(i) For all $a, b>0$ and $0<r<1$, we have

$$
m^{\pi_{r}}\left(a^{1 /(1-r)}, b^{1 /(1-r)}\right)=m^{\pi_{r}}\left(a^{r /(1-r)}, b^{r /(1-r)}\right) m(a, b)
$$

In particular (for $r=1 / 2)$, we obtain $m^{\pi}\left(a^{2}, b^{2}\right)=m^{\pi}(a, b) m(a, b)$.

(ii) Assume that $m$ is homogeneous and let $f$ and $f^{\pi_{r}}$ be the associated functions of $m$ and $m^{\pi_{r}}$, respectively. Then, for every $x>0$, one has

$$
f^{\pi_{r}}\left(x^{1 /(1-r)}\right)=f^{\pi_{r}}\left(x^{r /(1-r)}\right) f(x)
$$

In particular (for $r=1 / 2)$, we obtain $f^{\pi}\left(x^{2}\right)=f^{\pi}(x) f(x)$.

Proof

(i) By (8) we have successively

$$
\begin{aligned}
m^{\pi_{r}}\left(a^{1 /(1-r)}, b^{1 /(1-r)}\right) & =\prod_{n=1}^{\infty} m\left(a^{r^{n-1}}, b^{r^{n-1}}\right) \\
& =m(a, b) \prod_{n=2}^{\infty} m\left(a^{r^{n-1}}, b^{r^{n-1}}\right)=m(a, b) \prod_{n=1}^{\infty} m\left(a^{r^{n}}, b^{r^{n}}\right) .
\end{aligned}
$$

The desired result follows after a simple manipulation.

(ii) Follows from the fact that $m(a, b)=b f(a / b)$ when combined with (i). The proof is completed.

From the above proposition, we can derive some interesting results. The first result concerns an answer to the question that has been put in the above section. 
Corollary 3.1 Let $m$ be a symmetric and homogeneous mean such that $\left(m^{\pi_{k}}\right)_{k}$ is $p$ convergent. Then its limit is $m^{\pi_{\infty}}=G$, the geometric mean. In particular, $\left(L^{\pi_{k}}\right)_{k},\left(P^{\pi_{k}}\right)_{k}$, $\left(I^{\pi_{k}}\right)_{k}$ and $\left(A^{\pi_{k}}\right)_{k}$ are decreasingly $p$-convergent to the same limit $G$.

Proof If $\left(m^{\pi_{k}}\right)_{k}$ is $p$-convergent, then the sequence $\left(f^{\pi_{k}}\right)_{k}$ is also $p$-convergent, where $f^{\pi_{k}}$ is the associated function of $m^{\pi_{k}}$. That is, there exists a function (corresponding to a symmetric homogeneous mean) such that $g(x)=\lim _{k} f^{\pi_{k}}(x)$ for each $x>0$. According to Proposition 3.1(ii) and the recursive definition of $m^{\pi_{k}}$, we can write

$$
\forall x>0 \quad f^{\pi_{k}}\left(x^{2}\right)=f^{\pi_{k}}(x) f^{\pi_{k-1}}(x) .
$$

Then we deduce, by letting $k \rightarrow \infty$,

$$
\forall x>0 \quad g\left(x^{2}\right)=g(x) g(x)=(g(x))^{2} .
$$

It follows that $g(x)=\sqrt{x}$, which is the associated function of $G$, in this way proving the first part of the proposition. For the second part, as already pointed before, the sequences $\left(L^{\pi_{k}}\right)_{k},\left(P^{\pi_{k}}\right)_{k},\left(I^{\pi_{k}}\right)_{k}$ and $\left(A^{\pi_{k}}\right)_{k}$ are $p$-decreasing. It follows that they $p$-converge and by the first part they have $G$ as a common limit. The proof of the proposition is complete.

Corollary 3.2 Let $m_{1}$ and $m_{2}$ be two homogeneous means such that $m_{1}^{\pi_{r}}=m_{2}^{\pi_{r}}$ for a certain $r \in] 0,1\left[\right.$. Then $m_{1}=m_{2}$.

Proof Let $f$ and $g$ be the associated functions of $m_{1}$ and $m_{2}$, respectively. Assume that $m_{1}^{\pi_{r}}=m_{2}^{\pi_{r}}$ for some $\left.r \in\right] 0,1\left[\right.$, then $f^{\pi_{r}}(x)=g^{\pi_{r}}(x)$ for all $x>0$ and so

$$
f^{\pi_{r}}\left(x^{r /(1-r)}\right)=g^{\pi_{r}}\left(x^{r /(1-r)}\right)
$$

It follows that

$$
f^{\pi_{r}}\left(x^{r /(1-r)}\right) f(x)=g^{\pi_{r}}\left(x^{r /(1-r)}\right) f(x),
$$

and by Proposition 3.1(ii), we obtain

$$
f^{\pi_{r}}\left(x^{1 /(1-r)}\right)=g^{\pi_{r}}\left(x^{r /(1-r)}\right) f(x) .
$$

Since $f^{\pi_{r}}(x)=g^{\pi_{r}}(x)$ for all $x>0$, then

$$
g^{\pi_{r}}\left(x^{1 /(1-r)}\right)=g^{\pi_{r}}\left(x^{r /(1-r)}\right) f(x),
$$

or by Proposition 3.1(ii) again,

$$
g^{\pi_{r}}\left(x^{r /(1-r)}\right) g(x)=g^{\pi_{r}}\left(x^{r /(1-r)}\right) f(x) .
$$

We deduce that $f(x)=g(x)$ for each $x>0$ and so $m_{1}=m_{2}$, which completes the proof. 
Now, let us observe the next question: Does (9) (resp (10)) characterize $m^{\pi_{r}}$ for a given mean (resp. homogeneous mean) $m$ ? For the sake of simplicity, we assume that $m$ is homogeneous and we will prove the following theorem.

Theorem 3.1 Let $m$ be a homogeneous mean with its associated function $f$ and let $0<r<1$. Assume that there exists a continuous function $f_{r}$ such that $f_{r}(1)=1$ and

$$
\forall x>0 \quad f_{r}\left(x^{\alpha}\right)=f_{r}\left(x^{\alpha-1}\right) f(x), \quad \text { with } \alpha:=1 /(1-r)>1 .
$$

Then $f_{r}$ is the associated function of $m^{\pi_{r}}$ defined by (8).

Proof Assume that (11) holds. It is equivalent to

$$
\forall x>0 \quad f_{r}(x)=f_{r}\left(x^{r}\right) f\left(x^{1-r}\right)
$$

But we can apply this to write

$$
\forall x>0 \quad f_{r}\left(x^{r}\right)=f_{r}\left(x^{r^{2}}\right) f\left(x^{r-r^{2}}\right),
$$

which when substituted in (12) yields

$$
\forall x>0 \quad f_{r}(x)=f_{r}\left(x^{r^{2}}\right) f\left(x^{r(1-r)}\right) f\left(x^{1-r}\right) .
$$

By a simple mathematical induction, we can establish that for all integer $N \geq 1$ we have

$$
\forall x>0 \quad f_{r}(x)=f_{r}\left(x^{r^{N}}\right) \prod_{n=1}^{N} f\left(x^{(1-r) r^{n-1}}\right) .
$$

Since $0<r<1$, then $r^{N}$ tends to 0 when $N$ goes to $+\infty$. Letting $N \rightarrow+\infty$ in the previous equality, with the fact that $f_{r}$ is continuous and $f_{r}(1)=1$, we obtain

$$
f_{r}(x)=\prod_{n=1}^{\infty} f\left(x^{(1-r) r^{n-1}}\right),
$$

which, following Proposition 3.1(ii), is the associated function of $m^{\pi_{r}}$, in this way proving the desired result.

We now present some examples illustrating the above. In all these examples, $r$ and $\alpha$ are such that $0<r<1, \alpha:=1 /(1-r)>1$.

Example 3.1 Let $f(x)=\sqrt{x}$ be the associated function of $G$. We have $f\left(x^{\alpha}\right) / f\left(x^{\alpha-1}\right)=\sqrt{x}$, that is, $G^{\pi_{r}}=G$ for all $0<r<1$, which has been already pointed before.

Example 3.2 Let $f(x)=(x+1) / 2$ be the associated function of $A$. Clearly, we have

$$
\frac{f\left(x^{\alpha}\right)}{f\left(x^{\alpha-1}\right)}=\frac{x^{\alpha}+1}{x^{\alpha-1}+1}
$$


which is the associated function of $G_{\alpha-1, \alpha}$. Otherwise, $G_{\alpha-1, \alpha}^{\pi_{r}}=A$. In particular, with $r=1 / 2$ (and so $\alpha=2$ ), we obtain $C^{\pi}=A$. By Proposition 2.1, we deduce $C^{\pi} \pi=H$.

Example 3.3 Let $f(x)=(x-1) / \ln x, x>0$, with $f(1)=1$ be the associated function of $L$. A simple computation leads to

$$
\frac{f\left(x^{\alpha}\right)}{f\left(x^{\alpha-1}\right)}=\frac{\alpha-1}{\alpha} \frac{x^{\alpha}-1}{x^{\alpha-1}-1}
$$

which is the associated function of $E_{\alpha-1, \alpha}$, that is, $E_{\alpha-1, \alpha}^{\pi_{r}}=L$. In particular (if $r=1 / 2, \alpha=2$ ), we find $A^{\pi}=L$ and so $H^{\pi}=L^{*}$.

Example 3.4 Let $f(x)=e^{-1} x^{x /(x-1)}, x>0$, with $f(1)=1$ be the associated function of $I$. Similarly, we obtain

$$
\frac{f\left(x^{\alpha}\right)}{f\left(x^{\alpha-1}\right)}=\exp \left[\left(\frac{\alpha x^{\alpha}}{x^{\alpha}-1}-\frac{(\alpha-1) x^{\alpha-1}}{x^{\alpha-1}-1}\right) \ln x\right] .
$$

To find out if this latter function corresponds to a certain homogeneous mean for all $\alpha>1$ is left to the reader. For the particular case $r=1 / 2, \alpha=2$, the answer to this question is obviously positive since $f\left(x^{2}\right) / f(x)=x^{x /(x+1)}$ is the associated function of $S$. We then have $S^{\pi}=I$ and so $S^{*} \pi=I^{*}$.

Example 3.5 Let $f(x)=\frac{x-1}{2 \arcsin \frac{x-1}{x+1}}, x>0$, with $f(1)=1$ be the associated function of $P$. Obviously, we have

$$
\frac{f\left(x^{2}\right)}{f(x)}=(x+1) \frac{\arcsin \frac{x-1}{x+1}}{\arcsin \frac{x^{2}-1}{x^{2}+1}} .
$$

The fact that this latter function is the associated function of a certain homogeneous mean does not appear to be obvious. See the section below for a general point of view.

\section{Decomposable means}

For the sake of convenience, for concrete examples, we may introduce the following notion.

Definition 4.1 Let $m$ be a mean such that there exists a mean $m_{1}$ and some $\left.r \in\right] 0,1[$ satisfying $m=m_{1}^{\pi_{r}}$. Then we say that $m$ is $\left(m_{1}, r\right)$-decomposable. In the case $r=1 / 2$, we simply say $m$ is $m_{1}$-decomposable.

Proposition 4.1 With the above, the following properties are met:

(i) If $m$ is $\left(m_{1}, r\right)$-decomposable, then for all $s>0$, the generalized dual mean $m^{*}$ s is $\left(m_{1}^{\prime \prime}, r\right)$-decomposable.

(ii) If $m$ is $\left(m_{1}, r\right)$-decomposable and $m^{\prime}$ is $\left(m_{2}, r\right)$-decomposable, then for all $\alpha \in[0,1]$, $m^{1-\alpha} m^{\prime \alpha}$ is $\left(m_{1}^{1-\alpha} m_{2}^{\alpha}, r\right)$-decomposable.

Proof

(i) Comes from Proposition 2.1(ii).

(ii) It is an equivalent version of Proposition 2.1(iv). 
Now, we will illustrate the above notions and results by some examples.

Example 4.1 We have already seen that $G^{\pi_{r}}=G$ for each $\left.r \in\right] 0,1[$. We say that $G$ is selfdecomposable. Also, we can see that $\min ^{\pi_{r}}=\min$ and $\max ^{\pi_{r}}=\max$ for every $\left.r \in\right] 0,1[$.

Example 4.2 The relationship (1), written in a brief form $A^{\pi}=L$, says that $L$ is $A$ decomposable. By Proposition 4.1(i), we deduce that $L^{\prime \prime}$ is $H$-decomposable. We have also seen $S^{\pi}=I$, that is, $I$ is $S$-decomposable and so $I^{*}$ is $S^{*}$-decomposable. We leave it to the reader to see that $A$ is $C$-decomposable and so $H$ is $C^{*}$-decomposable. Other examples, in a more general point of view, will be stated below.

Example 4.3 In this example, we are interested in the link between two double-power means, namely the Stolarsky and Gini means. By virtue of this interest, we state its content as an explicit result from which we will derive some interesting consequences.

Theorem 4.1 For all real numbers $p$, $q$, the Stolarsky mean $E_{p, q}$ is $G_{p, q^{-}}$-decomposable, that is, the relationship

$$
E_{p, q}=\left(G_{p, q}\right)^{\pi}
$$

holds for all real numbers $p$ and $q$.

Proof Let

$$
f_{p, q}(x)=\left(\frac{x^{q}-1}{x^{p}-1}\right)^{1 /(q-p)}, \quad x>0, \text { with } f_{p, q}(1)=1
$$

be the associated function of $E_{p, q}$, with convenient forms for $p=q \neq 0$ and $p \neq 0, q=0$. Using Proposition 3.1, we obtain

$$
\frac{f_{p, q}^{\pi}\left(x^{2}\right)}{f_{p, q}^{\pi}(x)}=\left(\frac{x^{2 q}-1}{x^{2 p}-1} \cdot \frac{x^{p}-1}{x^{q}-1}\right)^{1 /(q-p)},
$$

which after simplification remains

$$
\frac{f_{p, q}^{\pi}\left(x^{2}\right)}{f_{p, q}^{\pi}(x)}=\left(\frac{x^{q}+1}{x^{p}+1}\right)^{1 /(q-p)}
$$

This latter function is that associated to $G_{p, q}$, that is, $E_{p, q}=G_{p, q}^{\pi}$. The proof is completed.

Corollary 4.1 The following chain of inequalities holds true:

$$
G^{\pi}=G<L^{\pi}<P^{\pi}<I^{\pi}<A^{\pi}=L<P<S^{\pi}=I<C^{\pi}=A<S<C .
$$

Proof According to the above theorem, we immediately deduce the following:

$$
A^{\pi}=L, \quad C^{\pi}=A, \quad G^{\pi}=G, \quad S^{\pi}=I .
$$


Since $G<L<P<I<A$, Proposition 2.1(iii) yields $G^{\pi}=G<L^{\pi}<P^{\pi}<I^{\pi}<A^{\pi}=L$. The desired inequalities follow by combining the two above chains of inequalities.

Another interesting consequence of the above theorem is that the increase monotonicity of $G_{p, q}$ with both $p, q$ implies that of $E_{p, q}$. In fact, by Proposition 2.1(iii) and Theorem 4.1, we successively obtain

$$
\begin{aligned}
\left(p_{1}<p_{2}, q_{1}<q_{2}\right) & \Longrightarrow G_{p_{1}, q_{1}}<G_{p_{2}, q_{2}} \quad \Longrightarrow \quad G_{p_{1}, q_{1}}^{\pi}<G_{p_{2}, q_{2}}^{\pi} \\
& \Longrightarrow E_{p_{1}, q_{1}}<E_{p_{2}, q_{2}}
\end{aligned}
$$

in this way proving the desired aim.

Theorem 4.1 contains more new applications: some extensions for $G_{p, q}$ can imply analogous ones for $E_{p, q}$. See Section 5 below for more details concerning this latter situation.

Example 4.4 Similarly to the above, we leave it to the reader to establish that $M_{p}^{\pi 3}=E_{p, 0}$, where $M_{p}$ refers to the Lehmer mean defined by

$$
M_{p}(a, b)=\left(\frac{a^{p}+a^{p / 2} b^{p / 2}+b^{p}}{3}\right)^{1 / p}
$$

with $M_{0}(a, b)=G(a, b)$. In particular, taking $p=1$, we obtain (another decomposition of $L)$ :

$$
L=\left(\frac{2}{3} A+\frac{1}{3} G\right)^{\pi_{3}} \text {. }
$$

We leave to the reader the routine task of formulating further examples in the aim to obtain some links between other special means.

Now, a question arises naturally from the above: Is it true that every mean is $\left(m_{1}, r\right)$ decomposable? In other words, let $m$ be a given mean, do a mean $m_{1}$ and a real number $0<r<1$ such that $m_{1}^{\pi_{r}}=m$ exist? The answer to this latter question appears to be interesting. In fact, for reason of simplicity, assume that $m$ is homogeneous and we search for a homogeneous mean $m_{1}$ such that $m_{1}^{\pi}=m$. According to Proposition 3.1, it is equivalent to have $f\left(x^{\alpha}\right)=f\left(x^{\alpha-1}\right) g(x)$ for all $x>0$, where $f$ denotes the associated function of the given mean $m$ and $g$ will be that of the unknown mean $m_{1}$. That is to say, the function $x \longmapsto f\left(x^{\alpha}\right) / f\left(x^{\alpha-1}\right)$ is the associated function to a certain mean $m_{1}$ for some $\alpha>1$. Combining this with Proposition 1.2, we can state the next result, which gives an answer to the above question.

Proposition 4.2 Let $m$ be a homogeneous mean with its associated function $f$. For some $0<r<1$, we put

$$
g_{r}(x)=f\left(x^{\alpha}\right) / f\left(x^{\alpha-1}\right), \quad \text { with } \alpha=1 /(1-r)>1 .
$$

Then the following assertions are equivalent:

(i) There exists a homogeneous mean $m_{1}$ such that $m$ is $\left(m_{1}, r\right)$-decomposable, that is, $m=m_{1}^{\pi}$. 
(ii) The function $x \longmapsto g_{r}(x)$ is the associated function of a certain homogeneous mean.

(iii) The following inequalities:

$$
f\left(x^{\alpha-1}\right) \leq f\left(x^{\alpha}\right) \leq x f\left(x^{\alpha-1}\right)
$$

hold for every $x \geq 1$, with reversed inequalities for each $x \leq 1$.

If in the above the function $x \longmapsto g_{r}(x)$ is increasing and $f$ is such that

$$
\forall x>0 \quad f\left(x^{\alpha}\right) f\left(x^{1-\alpha}\right)=x f\left(x^{-\alpha}\right) f\left(x^{\alpha-1}\right)
$$

then the homogeneous mean $m_{1}$ is symmetric and monotone.

The following corollary is immediate from the above proposition when combined with Proposition 1.2.

Corollary 4.2 Let $m$ be a homogeneous mean with its associated function $f$ and let us put $g(x)=f\left(x^{2}\right) / f(x)$. Then the following properties are equivalent:

(i) $m$ is $m_{1}$-decomposable.

(ii) $g$ is the associated function of a certain mean.

(iii) The double inequality

$$
f(x) \leq f\left(x^{2}\right) \leq x f(x)
$$

holds for all $x \geq 1$, with reversed inequalities for each $x \leq 1$.

If, moreover, $g$ is increasing and

$$
\forall x>0 \quad f\left(x^{2}\right) f(1 / x)=x f(x) f\left(1 / x^{2}\right)
$$

then $m_{1}$ is a symmetric and monotone mean.

Corollary 4.3 Let $m$ be a (symmetric) homogeneous monotone mean and let $0<r<1$ be a given real number. Then there exists a homogeneous mean $m_{1}$ such that $m$ is $\left(m_{1}, r\right)$ decomposable. In particular, every (symmetric) homogeneous monotone mean $m$ is $m_{1}$ decomposable for some homogeneous mean $m_{1}$.

Proof Let $f$ be the associated function of $m$ and set $\alpha=1 /(1-r)>1$. Since $m$ is monotone, then

$$
m(x, 1) \leq m\left(x^{\alpha}, 1\right) \leq m\left(x^{\alpha}, x\right)
$$

for all $x \geq 1$, with reversed inequalities for $x \leq 1$. It follows that

$$
f(x) \leq f\left(x^{\alpha}\right) \leq x f\left(x^{\alpha-1}\right)
$$

for every $x \geq 1$, with reversed inequalities if $x \leq 1$. By virtue of the above proposition, we can conclude the first part of the announced result. Taking $r=1 / 2, \alpha=2$, we obtain the second part and thus complete the proof. 
In the above corollary, we explicitly notice that the mean $m$ should be monotone in order to ensure that $m$ is $m_{1}$-decomposable for some mean $m_{1}$, but $m_{1}$ is not necessary monotone. As an example, we have already seen that $A$ is $C$-decomposable with $A$ monotone but $C$ is not monotone.

We can formulate the above in another way. Let $\mathcal{M}, \mathcal{M}_{h, m}, \mathcal{M}_{s, h, m}$ denote the set of means, homogeneous monotone means and symmetric homogeneous monotone means, respectively. For fixed $0<r<1$, let $\Phi_{r}: \mathcal{M} \longrightarrow \mathcal{M}$ be defined by $\Phi_{r}(m)=m^{\pi_{r}}$ for every mean $m$. Clearly, $\Phi_{r}(\mathcal{M})=\mathcal{D}_{r}$ is the set of $r$-decomposable means and the following meanchain of strict inclusions holds:

$$
\mathcal{M}_{s, h, m} \subset \mathcal{M}_{h, m} \subset \mathcal{D}_{r} \subset \mathcal{M}
$$

Example 4.5 The standard means $A, H, G, L, I$ are all (symmetric) homogeneous and monotone, then we find again that these means are decomposable. However, as already pointed before, the contraharmonic mean $C$ is not monotone and so we cannot apply the above corollary. Let us try to apply directly Proposition 4.2 . We can easily verify that the associated function $f(x)=\left(x^{2}+1\right) /(x+1)$ of $C$ does not satisfy (iii) and so $C$ is not decomposable.

We leave it to the reader to check if the means $S$ and $P$ are decomposable or not.

The reader can easily verify that all the above standard means $A, H, G, L, I, P, C, S$ satisfy relationship (15). However, for the corresponding functions $g$, it is not always monotone as in the case for $A$ and $H$. In the general case, the following result gives a sufficient condition for ensuring the increase monotonicity of $g$.

Proposition 4.3 Let $m$ be a symmetric homogeneous monotone mean and $f$ be its associated function. Suppose that the function $F(x)=f(x) / \sqrt{x}$ is strictly increasing. Then the function $g(x)=f\left(x^{2}\right) / f(x)$ will be strictly increasing.

Proof One has $g(x)=F\left(x^{2}\right) k(x)$, where $k(x)=x / f(x)$. Since $f(1 / x)=f(x) / x$ is decreasing, we get that $k$ is increasing. Since $F\left(x^{2}\right)$ is strictly increasing, we get that $g$ is strictly increasing (as a product of an increasing and a strictly increasing positive functions).

Example 4.6 The means $G$ and $L$ satisfy the conditions of the above proposition, whereas the means $A$ and $H$ do not. This rejoins the fact that $A$ is $C$-decomposable and $H$ is $C^{*}$ decomposable with $C$ and $C^{*}$ not monotone means. We leave it to the reader to verify if the means $I$ and $P$ satisfy or not the conditions of the above proposition.

\section{Application: means with several arguments}

In this section, we investigate an application of our above theoretical study. This application turns out the extension of some means from two variables to three or more arguments.

As is well known, the definition of a mean involving three or more arguments can be stated in a similar manner as that for bivariate mean. As special examples, the arithmetic, 
geometric and harmonic means with several variables are, respectively, given by

$$
\begin{aligned}
& A\left(a_{1}, a_{2}, \ldots, a_{k}\right)=\frac{\sum_{i=1}^{k} a_{i}}{k}, \\
& G\left(a_{1}, a_{2}, \ldots, a_{k}\right)=\sqrt[k]{\prod_{i=1}^{k} a_{i},} \quad H\left(a_{1}, a_{2}, \ldots, a_{k}\right)=\left(\frac{\sum_{i=1}^{k} a_{i}^{-1}}{k}\right)^{-1} .
\end{aligned}
$$

Extensions of the weighted geometric mean $S(a, b)$ and contraharmonic mean $C(a, b)$ are immediately given by

$$
\begin{aligned}
& S\left(a_{1}, a_{2}, \ldots, a_{k}\right)=\prod_{i=1}^{k} a_{i}^{a_{i} / S_{k}} \quad \text { with } S_{k}=\sum_{i=1}^{k} a_{i}, \\
& C\left(a_{1}, a_{2}, \ldots, a_{k}\right)=\frac{\sum_{i=1}^{k} a_{i}^{2}}{\sum_{i=1}^{k} a_{i}} .
\end{aligned}
$$

The following result is well known in the literature.

Proposition 5.1 The following inequalities:

$$
\begin{aligned}
H\left(a_{1}, a_{2}, \ldots, a_{k}\right) & <G\left(a_{1}, a_{2}, \ldots, a_{k}\right)<A\left(a_{1}, a_{2}, \ldots, a_{k}\right) \\
& <S\left(a_{1}, a_{2}, \ldots, a_{k}\right)<C\left(a_{1}, a_{2}, \ldots, a_{k}\right)
\end{aligned}
$$

hold true for all distinct real numbers $a_{1}, a_{2}, \ldots, a_{k}$.

However, the extension of the logarithm, identric and Seiffert means from two to three or more variables does not appear to be obvious from the above expressions of these means. In this sense, we refer the reader to [5, 8-10] for some extensions about the logarithmic and identric means. Here, we will derive other extensions of these latter means from our above study. In fact, the above transformation for means with two variables can be immediately stated in a similar manner for means involving several variables. For instance, we can define

$$
m^{\pi}\left(a_{1}, a_{2}, \ldots, a_{k}\right)=\prod_{n=1}^{\infty} m\left(a_{1}^{1 / 2^{n}}, a_{2}^{1 / 2^{n}}, \ldots, a_{k}^{1 / 2^{n}}\right) .
$$

It is also simple to see that $G^{\pi}\left(a_{1}, a_{2}, \ldots, a_{k}\right)=G\left(a_{1}, a_{2}, \ldots, a_{k}\right)$ for all $a_{1}, a_{2}, \ldots, a_{k}$.

The relationship $L=A^{\pi}$, which has been stated as a result for two variables, allows us to consider it as a definition for the logarithmic mean involving three or more arguments. That is, we can suggest that

$$
L\left(a_{1}, a_{2}, \ldots, a_{k}\right):=\prod_{n=1}^{\infty} A\left(a_{1}^{1 / 2^{n}}, a_{2}^{1 / 2^{n}}, \ldots, a_{k}^{1 / 2^{n}}\right)
$$

can be considered as a definition of the logarithmic mean with several variables. Now, the fact that if this definition coincides or not with some other ones as these given in $[8,10-$ 
12] seems to be an interesting problem. We omit the details about this latter point which is beyond our aim here.

Similarly, by virtue of the relation $I=S^{\pi}$, we can put a definition for identric mean with several variables

$$
I\left(a_{1}, a_{2}, \ldots, a_{k}\right)=\prod_{n=1}^{\infty} S\left(a_{1}^{1 / 2^{n}}, a_{2}^{1 / 2^{n}}, \ldots, a_{k}^{1 / 2^{n}}\right) .
$$

The comparison of this definition of $I\left(a_{1}, a_{2}, \ldots, a_{k}\right)$ with that given in $[9,10]$ appears also to be an interesting problem.

To give more justification for our above extensions, the following result, which extends the inequalities $G<L<I<A$ from two variables to several arguments, may be stated.

Proposition 5.2 With the above, the following inequalities:

$$
G\left(a_{1}, a_{2}, \ldots, a_{k}\right)<L\left(a_{1}, a_{2}, \ldots, a_{k}\right)<I\left(a_{1}, a_{2}, \ldots, a_{k}\right)<A\left(a_{1}, a_{2}, \ldots, a_{k}\right)
$$

hold for all distinct real numbers $a_{1}, a_{2}, \ldots, a_{k}$.

Proof For the sake of simplicity, we write (16) in a brief form $G<A<S<C$. According to (17), we easily show that $G^{\pi}<A^{\pi}<S^{\pi}<C^{\pi}$. Since $G^{\pi}=G, A^{\pi}=L, S^{\pi}=I$, we then obtain $G<L<I<C^{\pi}$. Using (17) again, a simple computation yields $C^{\pi}=A$. The proof is complete.

More generally, the relationship $E_{p, q}=G_{p, q}^{\pi}$ appears to be a good tool for extending the Stolarsky mean $E_{p, q}(a, b)$ from two to three or more arguments. In fact, the Gini mean $G_{p, q}(a, b)$ seems simple to extend for several arguments as well:

$$
G_{p, q}\left(a_{1}, a_{2}, \ldots, a_{k}\right)=\left(\frac{\sum_{i=1}^{k} a_{i}^{q}}{\sum_{i=1}^{k} a_{i}^{p}}\right)^{1 /(q-p)}
$$

with the convenient cases

$$
G_{p, p}\left(a_{1}, a_{2}, \ldots, a_{k}\right)=\exp \left(\frac{\sum_{i=1}^{k} a_{i}^{p} \ln a_{i}}{\sum_{i=1}^{k} a_{i}^{p}}\right) \text { and } G_{0,0}\left(a_{1}, a_{2}, \ldots, a_{k}\right)=G\left(a_{1}, a_{2}, \ldots, a_{k}\right)
$$

With this, we can suggest that the Stolarsky mean with several variables can be defined by

$$
E_{p, q}\left(a_{1}, a_{2}, \ldots, a_{k}\right)=\prod_{n=1}^{\infty} G_{p, q}\left(a_{1}^{1 / 2^{n}}, a_{2}^{1 / 2^{n}}, \ldots, a_{k}^{1 / 2^{n}}\right) .
$$

Now, the question concerning the comparison of this definition of $E_{p, q}\left(a_{1}, a_{2}, \ldots, a_{k}\right)$ with some other ones given in $[13,14]$ can be considered as an interesting purpose for future research. 
Authors' contributions

Both authors jointly worked, read and approved the final version of the paper.

\section{Author details}

${ }^{1}$ Faculty of Science, Department of Mathematics, Taibah University, P.O. Box 30097, Al Madinah Al Munawwarah, Zip Code 41477, Kingdom of Saudi Arabia. ${ }^{2}$ Faculty of Sciences, Department of Mathematics, Moulay Ismail University, Meknes, Morocco. ${ }^{3}$ Department of Mathematics, Babes-Bolyai University, Str. Kogalniceanu nr.1, Cluj-Napoca, 400084, Romania.

\section{Received: 25 August 2012 Accepted: 17 January 2013 Published: 5 March 2013}

\section{References}

1. Bullen, PS: Handbook of Means and Their Inequalities, 2 nd edn. Mathematics and Its Applications. Springer, Berlin (1987)

2. Matkowski, J: Convex functions with respect to a mean and a characterization a quasi-arithmetic mean. Real Anal. Exch. 29(1), 220-246 (2003/2004)

3. Matkowski, J: On weighted extension of Cauchy's means. J. Math. Anal. Appl. 319, $215-227$ (2006)

4. Sándor, J: On the identric and logarithmic means. Aequ. Math. 40, 261-270 (1990)

5. Matkowski, J: Iterations of the mean-type mappings. In: Sharkovsky, AN, Susko, IM (eds.) ECIT'08. Grazer Math. Ber. vol. 354, pp. 158-179 (2009)

6. Raïssouli, M: Approaching the power logarithmic and difference means by algorithms involving the power binomial mean. Int. J. Math. Math. Sci. 2011, Article ID 687825 (2011)

7. Raïssouli, M: Stabilizability of the Stolarsky mean and its approximation in terms of the power binomial mean. Int. J. Math. Anal. 6(18), 871-881 (2012)

8. Mustonen, S: Logarithmic mean for several arguments. http://www.survo.fi/papers/logmean.pdf (2002)

9. Sándor, J, Trif, T: A new refinement of the Ky Fan inequality. Math. Inequal. Appl. 2(4), 529-533 (1999)

10. Xiao, Z-G, Zhang, Z-H: The inequalities $G \leq L \leq I \leq A$ in $n$ variables. JIPAM. J. Inequal. Pure Appl. Math. 4, Article ID 39 (2003)

11. Pittenger, AO: The logarithm mean in $n$ variables. Am. Math. Mon. 92, 99-104 (1985)

12. Raïssouli, M: Logarithmic functional mean in convex analysis. JIPAM. J. Inequal. Pure Appl. Math. 10(4), Article ID 102 (2009)

13. Neuman, E: Stolarsky means of several variables. JIPAM. J. Inequal. Pure Appl. Math. 6(2), Article ID 30 (2005)

14. Pečarić, J, Šimić, V: Stolarsky-Tobey mean in $n$ variables. Math. Inequal. Appl. 2, 325-341 (1999)

doi:10.1186/1029-242X-2013-89

Cite this article as: Raïssouli and Sándor: On a method of construction of new means with applications. Journal of Inequalities and Applications 2013 2013:89.

\section{Submit your manuscript to a SpringerOpen ${ }^{\circ}$ journal and benefit from:}

- Convenient online submission

- Rigorous peer review

- Immediate publication on acceptance

- Open access: articles freely available online

- High visibility within the field

- Retaining the copyright to your article 